\title{
Mesenchymal stem cells induce dendritic cell immune tolerance via paracrine hepatocyte growth factor to alleviate acute lung injury
}

Zhonghua Lu, Wei Chang, Shanshan Meng, Xiuping Xu, Jianfeng Xie, Fengmei Guo, Yi Yang, Haibo Qiu and Ling Liu ${ }^{*}$

\begin{abstract}
Background: Mesenchymal stem cells (MSCS) have been shown to alleviate acute lung injury (ALI) via paracrine hepatocyte growth factor (HGF) and to induce the differentiation of dendritic cells (DCs) into tolerogenic dendritic cells (DCregs) and participate in the immune response. However, whether MSCs induce the production of DCregs by secreting HGF to alleviate early ALI remains unclear. We observed that the protective effect of mouse bone marrow-derived MSCs against lipopolysaccharide (LPS)-induced ALI was achieved by inducing mature DCs (mDCs) to differentiate into DCregs, and its mechanism is related to the activation of the HGF/Akt pathway.
\end{abstract}

Methods: MSCs or MSCs with overexpression or knockdown of HGF were cocultured with DCs derived from mouse bone marrow using a Transwell system for 3 days. Moreover, we used MSCs or MSCs with overexpression or knockdown of HGF to treat LPS-induced ALI mice for $24 \mathrm{~h}$. Flow cytometry was performed to measure the phagocytosis, accumulation, and maturation of DCs, as well as proliferation of T cells. Lung injury was estimated by lung wet weight to body weight ratio (LWW/BW) and histopathological analysis. Furthermore, we used the Akt inhibitor MK-2206 in a coculture system to elucidate the role of the HGF/Akt pathway in regulating the differentiation of DCs into regulatory DCs and relieving lung injury in early ALI mice.

Results: Immature DCs (imDCs) were induced to mature after $24 \mathrm{~h}$ of LPS (50 $\mathrm{ng} / \mathrm{ml})$ stimulation. MSCs or HGF induced the differentiation of mDCs into regulatory DCs characterized by low expression of MHCII, CD86, and CD40 molecules, strong phagocytic function, and the ability to inhibit T cell proliferation. The effect of MSCs on DCregs was enhanced with the increase in HGF secretion and was weakened with the decrease in HGF secretion. DCregs induced by recombinant HGF were attenuated by the Akt inhibitor MK-2206. Lung DC aggregation and mDC ratio increased in LPS-induced ALI mice, while treatment with MSCs decreased lung DC aggregation and maturation and alleviated lung pathological injury. High expression of the HGF gene enhanced the above effect of MSCs, while decreased expression of HGF weakened the above effect of MSCs.

Conclusions: MSCs alleviate early ALI via paracrine HGF by inducing mDCs to differentiate into regulatory DCs. Furthermore, the mechanism of HGF-induced differentiation of mDCs into DCregs is related to the activation of the Akt pathway.

Keywords: Acute respiratory distress syndrome (ARDS), Acute lung injury (ALI), Dendritic cells, Hepatocyte growth factor (HGF), Mesenchymal stem cell (MSC), Akt

\footnotetext{
* Correspondence: liulingdoctor@126.com

Department of Critical Care Medicine, Zhongda Hospital, School of Medicine,

Southeast University, 87 Dingjia Bridge, Hunan Road, Gu Lou District, Nanjing 210009, China
}

(c) The Author(s). 2019 Open Access This article is distributed under the terms of the Creative Commons Attribution 4.0 International License (http://creativecommons.org/licenses/by/4.0/), which permits unrestricted use, distribution, and reproduction in any medium, provided you give appropriate credit to the original author(s) and the source, provide a link to the Creative Commons license, and indicate if changes were made. The Creative Commons Public Domain Dedication waiver (http://creativecommons.org/publicdomain/zero/1.0/) applies to the data made available in this article, unless otherwise stated. 


\section{Background}

At present, acute respiratory distress syndrome (ARDS) remains a relatively common and lethal or disabling syndrome despite decades of improvements in supportive and pharmacological interventions [1-4]. However, there may be heterogeneity of disease mechanisms in ARDS due to different causes, possibly contributing to the failure of countless interventions to improve outcomes in clinical trials [5]. Compared with extrapulmonary ARDS, pathological inflammatory infiltration and inflammatory markers of pulmonary ARDS are more obvious and more sensitive to treatment response [6]. An uncontrolled inflammatory response is the key mechanism of pulmonary ARDS pathogenesis [7]. Innate immune cellmediated damage to the alveolar endothelial and epithelial barrier, resulting in increased accumulation of protein-rich edema fluid in the interstitium and alveoli, is the initial response of the lungs to injury in the exudation phase of ARDS [8-10]. Dendritic cells (DCs) are the most important antigen-presenting cells and play a key role in the initiation of primary immune responses and the induction of tolerance in the pulmonary immune response network [11-13].

Previous studies have shown that the number and maturity of conventional DCs (cDCs) in the lungs of ALI mice are significantly increased, and promoting the maturation of pulmonary DCs can aggravate pulmonary inflammatory reactions and pathological injury [14-16]. Therefore, mature DCs (mDCs) play an important role in the pathophysiology of early ALI, and regulating the immune function of mDCs may have clinical significance in the treatment of ARDS.

Under certain conditions (such as drugs, soluble cytokines, stem cells, and changes in the local microenvironment), $\mathrm{mDCs}$ can be induced into regulatory $\mathrm{DCs}$ (DCregs), whose main role is to maintain immune tolerance and negatively regulate the effects of the immune response. Recently, studies have shown that mesenchymal stem cells (MSCs) have a superior immunomodulatory capacity to induce the differentiation of DCs into DCregs [17-21], which have a stable phenotype even under lipopolysaccharide (LPS) stimulation [19]. A growing number of studies have also provided convincing data on the beneficial effects of MSCs in treating LPS-induced acute lung injury (ALI) [22-25]. However, it is not clear whether MSCs improve the early lung injury of ALI by inducing $\mathrm{mDCs}$ to differentiate into DCregs.

Our previous studies provided reliable data on the protective effects of hepatocyte growth factor (HGF) secreted by MSCs on ALI in vitro and in vivo [26, 27]. Several studies have shown that HGF-treated DCs were characterized by increased expression of programmed death ligand 1 (PD-L1) and the ability to promote the development of IL-10-secreting regulatory $\mathrm{T}$ cells [28].
HGF also inhibits DC migration by binding to the HGF receptor-mesenchymal transition factor (c-Met) on the surface of DCs [29], which promotes downstream activation of the phosphatidylinositol 3-kinase/Akt pathway, inhibits antigen presentation, and downregulates surface markers for T cell activation [30, 31]. Whether the Akt pathway is an important link in HGF-induced differentiation of $\mathrm{mDCs}$ into DCregs remains unknown.

The aim of this study was to determine whether the protective effect of MSCs against LPS-induced ALI was achieved by inducing mDCs to differentiate into DCregs and whether the mechanism is related to the activation of the HGF/Akt pathway. We used coculture experiments to test the phenotype, phagocytosis, stimulation of $\mathrm{T}$ cell proliferation, and cytokine secretion of DCs. To investigate the effects and potential mechanisms of MSCs inducing mDCs to convert into DCregs, we used MSCs with low expression (shHGF-MSCs) and overexpression (HGF-MSCs) of HGF genes cocultured with $\mathrm{mDCs}$ and then explored the potential mechanisms of the protective effects of $\mathrm{HGF}$ on inducing $\mathrm{mDCs}$ to convert into DCregs by the Akt inhibitor MK2206. To study the effect of HGF in MSCs in the ALI mouse model, we used HGF-MSCs and shHGF-MSCs to treat LPSinduced ALI mice and evaluate lung pathological injury, DC phenotype, pulmonary edema, etc. This study provides an immunological explanation for the reduction of LPS-induced lung injury by MSCs via paracrine HGF.

\section{Methods}

Mice

Specific-pathogen-free male BALB/c and C57BL/6 mice (age 6-8 weeks, weight 20-25 g) were purchased from the Laboratory Animal Center of Yangzhou University (Yangzhou, China). All animal experiments were carried out in accordance with the National Institutes of Health Guide for the Care and Use of Laboratory Animals. All of the experimental procedures were approved by the Southeast University Ethics Committee.

\section{MSC culture}

Mouse MSCs and DCs were used in the present study. MSCs were purchased from Cyagen Biosciences, Inc. (Guangzhou, China). The supplier identified MSCs based on the cell surface phenotype and pluripotency. Fluoresceinconjugated monoclonal antibodies, including anti-CD29, anti-CD44, anti-CD117, anti-Sca-1, anti-CD31, and anti$\mathrm{CD} 45$, were used. The multipotent potential of MSCs for differentiation along the adipogenic, osteogenic, and chondrogenic lineages was determined by staining with Oil Red $\mathrm{O}$, Alizarin red, and toluidine blue, respectively, followed by culture in adipogenic, osteogenic, and chondrogenic differentiation media, respectively (Cyagen Biosciences, Inc.) for 2 to 3 weeks, thus verifying their identity as mouse MSCs. 
MSCs were cultured in DMEM/F12 (Wisent, Nanjing, China) containing $10 \%$ fetal bovine serum (FBS) (Wisent, Nanjing, China) and grown in a humidified 5\% CO2 incubator at $37^{\circ} \mathrm{C}$.

\section{Generation of mouse BM-derived DCs}

Bone marrow (BM)-derived DCs were generated as previously described with minor modifications [32]. BM cells were extracted from the medullary cavity of the femur and tibia. The erythrocytes were lysed using Lysing Buffer (BD Pharm Lyse ${ }^{\mathrm{mx}}$, USA), washed three times in PBS, and cultured in 100-mm dishes with $2 \times 10^{6}$ cells containing RPMI-1640 (Wisent, Nanjing, China) medium supplemented with 10\% FBS (Wisent, Nanjing, China), $40 \mathrm{ng} / \mathrm{ml}$ recombinant murine granulocyte-macrophage colonystimulating factor (GM-CSF; NOVUS), and $40 \mathrm{ng} / \mathrm{ml}$ recombinant murine interleukin-4 (IL-4; NOVUS) in a humidified $5 \% \mathrm{CO} 2$ incubator at $37^{\circ} \mathrm{C}$. For the isolation of imDCs, non-adherent cells were gently washed out on day 3 , and the remaining loosely adherent cell clusters were collected and purified by anti-CD11c micromagnetic beads (Miltenyi Biotec) on day 6. Purified imDCs cultured for an additional $24 \mathrm{~h}$ under the stimulation of $50 \mathrm{ng} / \mathrm{ml}$ bacterial lipopolysaccharide (LPS; Sigma-Aldrich) were used as mDCs. The purity of the cells was greater than $90 \%$. Cytofluorimetric analysis was performed to evaluate the DC maturation phenotype (CD40, CD86, and MHCII).

\section{Reagent treatments}

Before some experiments, purified imDCs cultured for an additional $24 \mathrm{~h}$ under the stimulation of $50 \mathrm{ng} / \mathrm{ml}$ bacterial LPS (Sigma-Aldrich) were used as mDCs. To determine the roles and mechanisms of $\mathrm{HGF}$, we introduced recombinant murine HGF $(50 \mathrm{ng} / \mathrm{ml}$, R\&D Systems, USA) into mature dendritic cells (mDCs) containing 5\% FCS and $20 \mathrm{ng} / \mathrm{ml}$ GM-CSF RPMI-1640 medium. In addition, phosphatebuffered saline (PBS) was used as a negative control, and the Akt inhibitor MK-2206 (5 $\mu \mathrm{mol} / \mathrm{l}$, Selleck) was used to inhibit the activation of the Akt pathway $3 \mathrm{~h}$ before HGF treatment of $\mathrm{mDC}$. The drug concentrations used were according to our preliminary experiments.

\section{Production of lentiviral vectors and transduction of MSCs} MSCs and 293FT cells were purchased from Cyagen Biosciences, Inc. (Guangzhou, China) as previously described. MSCs from passages 4-7 were used for transduction and HGF gene overexpression and knockdown experiments. HGF gene overexpression and reduced expression were achieved using lentiviral vectors, and lentiviruses for overexpression and reduced expression specific for enhanced green fluorescent protein (EGFP) (PDS087 and PDS019) were used as negative controls. The lentiviruses were packaged in 293T cells (Cyagen Biosciences, Inc.) with the aid of three packaging plasmids, and then a higher or lower lentivirus titer was obtained. The MSCs were transfected and screened by the antibiotic blasticidin for 7 to 14 days. Subsequently, MSCs carrying empty vectors and EGFP (NC-HGF-MSCs, NC-shHGF-MSCs) or MSCs carrying both the HGF gene and EGFP (HGF-MSCs, shHGF-MSCs) were harvested.

\section{DC and MSC coculture}

MSCs were seeded onto the lower chamber of a six-well Transwell system (0.4-mm pore size membrane; Corning, Cambridge, MA, USA). When MSCs, NC-HGF-MSCs, HGF-MSCs, NC-shHGF-MSCs, and shHGF-MSCs were attached, the medium was changed with RPMI-1640 medium containing 5\% FCS (Wisent, Inc., Nanjing, China) and $20 \mathrm{ng} / \mathrm{ml} \mathrm{GM-CSF}, 5 \times 105 \mathrm{mDCs}$ were seeded onto the upper chamber per well in six-well plates. The ratio of MSCs $/ \mathrm{mDCs}$ is $10: 1$. After cocultivation for $72 \mathrm{~h}$, the upper chamber cells were taken for examination or applied to the next experiment.

\section{ALI model}

The ALI model was induced as previously described with minor modifications [33]. Briefly, mice were intraperitoneally injected with $50 \mathrm{mg} / \mathrm{kg}$ pentobarbital. LPS ( $5 \mathrm{mg} / \mathrm{kg}$ ) (Sigma-Aldrich) was delivered to the lungs through a tracheostomy, and the incision was sewed up. Mice were returned to the cage until fully awake.

\section{Experimental groups and sample acquisition}

The mice were randomly assigned to one of the following groups ( $n=5$ mice per group): control group (Con), mice were given the same amount of $0.9 \%$ normal saline (NS) or PBS at the corresponding time; ALI group (ALI), mice received $5 \mathrm{mg} / \mathrm{kg}$ LPS to establish the ALI model; MSC + ALI group (MSCs), mice received MSCs $(500,000$ cells in $150 \mu \mathrm{l}$ PBS) via the tail vein $6 \mathrm{~h}$ after LPS; NC-HGFMSC + ALI group (NC-HGF-MSCs), mice received NCHGF-MSCs (500,000 cells in $150 \mu \mathrm{l}$ PBS) via the tail vein $6 \mathrm{~h}$ after LPS; HGF-MSC + ALI group (HGF-MSCs), mice received HGF-MSCs $(500,000$ cells in $150 \mu \mathrm{PBS})$ via the tail vein $6 \mathrm{~h}$ after LPS; NC-shHGF-MSC + ALI group (NC-shHGF-MSCs), mice received NC-shHGF-MSCs (500,000 cells in $150 \mu \mathrm{l}$ PBS) via the tail vein $6 \mathrm{~h}$ after LPS; shHGF-MSC + ALI group (shHGF-MSCs), mice received shHGF-MSCs $(500,000$ cells in $150 \mu \mathrm{l}$ PBS) via the tail vein $6 \mathrm{~h}$ after LPS. The mice were sacrificed after $24 \mathrm{~h}$; lung tissue was collected for single cell isolation and histological examination in accordance with slightly modified previous methods [14].

\section{Evaluation of lung edema}

Lung wet weight to body weight ratios (LWW/BW), which reflect the severity of lung vascular permeability and lung edema, were obtained for the control, ALI, 
MSC, NC-HGF-MSC, HGF-MSC, NC-shHGF-MSC, and shHGF-MSC groups.

\section{Lung histopathology}

The right upper lobe was embedded in paraffin and sagittally sliced at 5- $\mu \mathrm{m}$ thickness. The sections were stained with hematoxylin and eosin. Edema, alveolar and interstitial inflammation and hemorrhage, atelectasis, necrosis, and hyaline membrane formation were each scored using a 0 to 4-point scale. The severity of lung injury was calculated as the sum of the scores as previously described [26].

\section{Flow cytometry}

Lung cell isolation and the measurement of the accumulation and maturation of DCs by flow cytometry were performed as previously described [16]. For phenotypic analysis of cell surface marker expression, cells were harvested, resuspended in PBS, incubated for 15 min with FcR blocking reagent, and then incubated for $15 \mathrm{~min}$ with PE-, APC-, PE-Cy7-, PerCP-, or FITC-conjugated monoclonal antibodies (mAbs) on ice. DCs were stained with antibodies against CD11c, CD40, CD86, CD11b, MHCII (Miltenyi Biotec, Bergisch Gladbach, Germany), PD-L1 (BD Pharmingen), and IL-27 (IL-27p28; BioLegend, USA). Mouse IgG1 isotype control antibodies were used in parallel as negative controls. Carboxyfluorescein diacetate succinimidyl ester (CFSE) (BD Pharmingen) was used in $\mathrm{T}$ cells, and FITC-dextran was used to examine the phagocytosis of DCs. The stained cells were washed twice and resuspended in cold buffer and then analyzed by flow cytometry (FACSCalibur; NovoCyte), and the results were processed using NovoExpress software. The results are expressed as the percentage of positively stained cells relative to the total cell number.

\section{Cytokine analysis}

DCs were separated and washed after coculture with MSCs for $72 \mathrm{~h}$ and then cultured for $24 \mathrm{~h}$. The supernatant was collected, and the concentration of interleukin-12 (IL-12), transforming growth factor beta (TGF- $\beta$ ), and interleukin10 (IL-10) secreted by DCs was determined by enzymelinked immunosorbent assay (ELISA, RayBiotech). Quantitative analysis of HGF was performed on supernatants derived from MSC cultures by ELISA according to the manufacturer's instructions (ELISA, RayBiotech). HGF concentrations were determined using a standard curve constructed by titration of the HGF standard.

\section{Endocytosis assay}

To determine the phagocytic capacity of $\mathrm{DC}$, imDC, $\mathrm{mDC}$, HGF-DC, MSC-DC, NC-HGF-MSC-DC, NC-shHGF-MSCDC, HGF-MSC-DC, or shHGF-MSC-DC was incubated at $37^{\circ} \mathrm{C}$ or at $4{ }^{\circ} \mathrm{C}$ as a negative control for $4 \mathrm{~h}$ with FITC- conjugated OVA (FITC-OVA; AnaSpec) at a final concentration of $100 \mathrm{ng} / \mathrm{ml}$ containing RPMI-1640 with $10 \%$ FCS, washed twice with ice-cold PBS containing $0.5 \%$ bovine serum albumin (BSA; BioFroxx), and then resuspended in cooled PBS for immediate flow cytometry.

\section{Mitogen proliferative assay}

CFSE-labeled solenocytes $\left(5 \times 10^{5}\right.$ cells/well $)$ were incubated with $5 \mu \mathrm{g} / \mathrm{ml}$ concanavalin A (ConA; Sigma-Aldrich) or cocultured with allogenic DCs (mDCs, MSC-DCs, NCHGF-MSC-DCs, NC-shHGF-MSC-DCs, HGF-MSC-DCs, or shHGF-MSC-DCs, $5 \times 10^{4}$ cells/well) in a total volume of $0.2 \mathrm{ml}$ medium in $96-$-well U-bottom plates.

\section{Western blot analysis}

For the HGF/c-Met/Akt assay, phosphorylated Akt, total Akt, and phosphorylated or total c-Met were measured by Western blot analysis. Proteins were separated by sodium dodecyl sulfate-polyacrylamide gel electrophoresis and transferred to polyvinylidene difluoride (PVDF) membranes. The membranes were incubated with primary antibodies against c-Met, HGF (1:1000; Abcam), phospho-c-Met, Akt, and phospho-Akt (1:1000; Cell Signaling) at a $1: 1000$ dilution at $4{ }^{\circ} \mathrm{C}$ overnight. The membranes were incubated with secondary antibody for $1 \mathrm{~h}$ at room temperature. Immunoblots were visualized using enhanced chemiluminescence (ECL; Thermo Scientific). The expression levels from whole cell extract were normalized against that of $\beta$-actin.

\section{Statistical analysis}

All statistical analyses were performed using SPSS 23.0 software and GraphPad Prism 7.0. One-way analysis of variance (ANOVA) or two-tailed Student's $t$ test was used to determine the significance between the groups. Data are expressed as the mean \pm standard deviation (SD). $P<0.05$ was considered significant.

\section{Results \\ LPS induces the differentiation of imDCs into $\mathrm{mDCs}$}

Mouse BM-derived DCs showed typical characteristics of DCs on day 3, becoming clustered adherent cells and showing various protruding veils, and the typical DC traits became more apparent on the 7 th day (Fig. 1a). CD11c ${ }^{+}$ DCs reached over $90 \%$ purity after magnetic bead sorting. imDCs were treated with LPS $(0-1000 \mathrm{ng} / \mathrm{ml})$ for 0,24 , and $48 \mathrm{~h}$. The LPS-induced $\mathrm{mDC}$ phenotype marked by the expression of MHCII, CD86, and CD40 was positively dose-dependent when LPS concentrations were below $50 \mathrm{ng} / \mathrm{ml}$ (Fig. 1b, c), but the percentage of cells expressing the mature phenotype was highest at $24 \mathrm{~h}$ (Fig. 1d, e). imDCs were induced to mature after $24 \mathrm{~h}$ of $50 \mathrm{ng} / \mathrm{ml}$ LPS stimulation. 
A

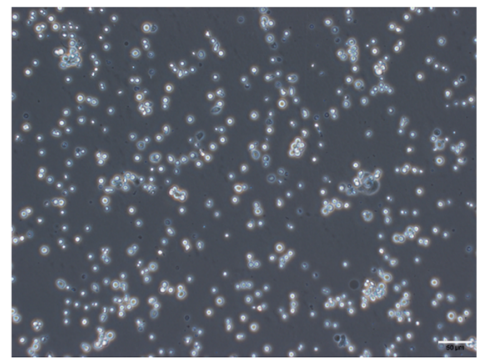

A:×200 ( Day1 )

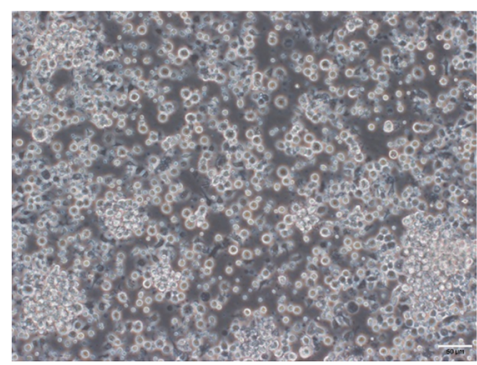

B:×200 ( Day3 )

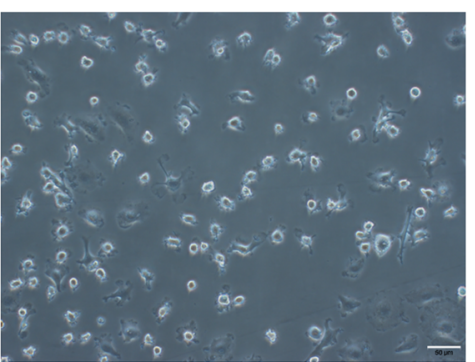

C:×200 ( Day7 )

B
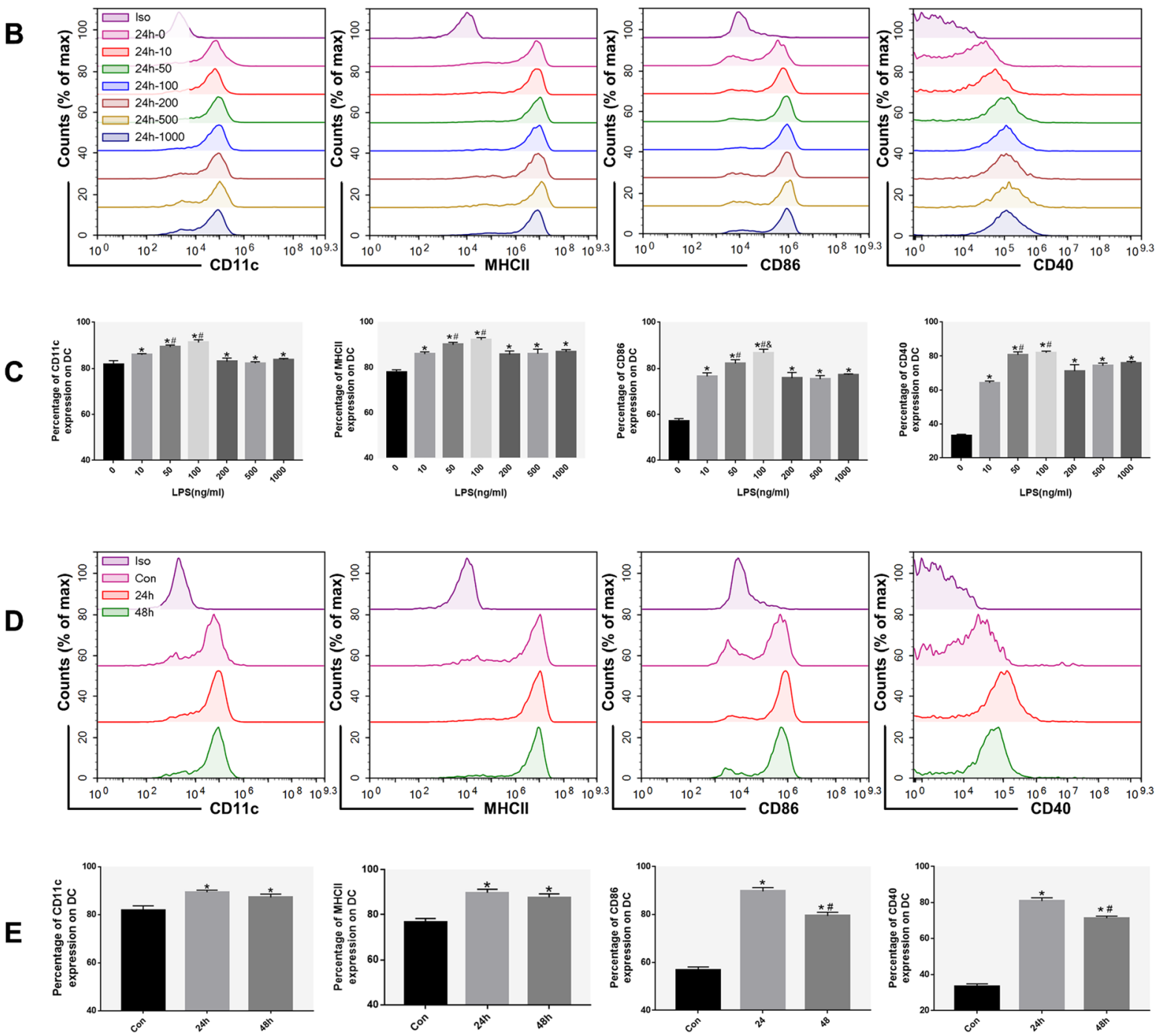

Fig. 1 (See legend on next page.) 
(See figure on previous page.)

Fig. 1 Induction and identification of DCs. a The morphology of DCs. Cell morphology on days 1, 3 (left and middle, monocytes in the presence of GM-CSF and IL-4), and 7 (right, imDC cultured for $24 \mathrm{~h}$ under LPS stimulation) (×200 magnification). b Immunophenotype analysis of DCs (expression of MHCII, CD86, and CD40 in DCs cultured for $24 \mathrm{~h}$ in the presence of LPS at concentrations ranging from 0 to $1000 \mathrm{ng} / \mathrm{ml}$ ). c The percentage of DCs positive for MHCII, CD86, and CD40 after incubation for $24 \mathrm{~h}$ with LPS at concentrations ranging from 0 to $1000 \mathrm{ng} / \mathrm{ml}$. $\mathbf{d}$ Immunophenotype analysis of DCs (expression of MHCII, CD86, and CD40 on DCs after culture for $0 \mathrm{~h}, 24 \mathrm{~h}$, and $48 \mathrm{~h}$ with an LPS concentration of $50 \mathrm{ng} / \mathrm{ml}$ ). e The percentage of DCs expressing MHCll, CD86, and CD40 after $0 \mathrm{~h}, 24 \mathrm{~h}$, and $48 \mathrm{~h}$ cultured at an LPS concentration of $50 \mathrm{ng} / \mathrm{ml}$. $n=3$. $\mathbf{c} * P<0.05$ versus LPS $0 \mathrm{ng} / \mathrm{ml}$ group; $\# P<0.05$ versus LPS $10 \mathrm{ng} / \mathrm{ml}$ group; $\& P<0.05$ versus LPS $50 \mathrm{ng} / \mathrm{ml}$ group. e ${ }^{*} P<0.05$ versus Con group; $\# P<0.05$ versus $24 \mathrm{~h}$ group. Data are expressed as mean \pm SD. Each experiment was repeated three times

\section{MSCs and rhHGF induce mDCs to convert into DCregs}

Interestingly, in contrast to the expression levels in mDCs, phenotype analysis (Fig. 2a) showed that MSCor rhHGF-treated $\mathrm{mDCs}$ expressed less functional markers, such as MHCII, CD86, and CD40, and were similar to imDCs. However, in contrast to imDCs, the addition of LPS to these cells could not restore the expression of the above functional markers, indicating the MSCs-induced mDCs to differentiate into a novel DC population (regulatory DCs) with a more stable phenotype than imDCs. Additionally, compared to $\mathrm{mDCs}$, these novel DCs exhibited stronger phagocytic capacity similar to imDCs (Fig. 2b). We also investigated whether MSC-DCs had an immunomodulatory capacity. When CFSE-labeled splenocytes, used as responders, were cocultured with mDCs, MSC-DCs, and rhHGF-DCs, MSC-DCs and rhHGF-DCs had the weakest effect on stimulating lymphocyte activation (Fig. 2c). Furthermore, MSC- or HGF-induced DCs expressed more immunosuppressive molecules PD-L1 and IL-27 than mDCs (Fig. 2d). Moreover, after culturing DCs independently for another $24 \mathrm{~h}$ after thorough washes, we observed that IL-10 and TGF- $\beta$ were increased in the MSC-DC and rhHGF-DC groups, and IL-12 was decreased (Fig. 2e). In the process of MSC- or rhHGF-induced $\mathrm{mDC}$ immune tolerance, the phosphorylation of the HGF-specific receptor c-Met protein was significantly increased (Fig. 2f). The results showed that the immunomodulatory properties of MSC-regulated DCs may depend on paracrine HGF in vitro.

\section{HGF expression in genetically modified MSCs}

Our previous research shows that changes in HGF secreted by MSCs have been observed [34]. Whether MSCs induce mDCs to convert into DCregs by secreting HGF remains unclear. To further determine whether the inhibitory effect of MSC-DCs was mediated by secreting HGF, HGF was overexpressed or knocked down by transducing MSCs with HGF or RNAi retroviral vectors. Fluorescence microscopy (Fig. 3a) and flow cytometry (Fig. 3b) images showed the expression of EGFP. The transduction efficiency was over $90 \%$ and was well maintained over 20 passages. The expression of HGF mRNA in the HGF-MSC group was approximately 12-fold greater than that of the MSC group, which was fivefold higher than that in the knockdown group (Fig. 3c). HGF protein expression was also increased in the HGF-MSC group while it was decreased in the shHGF-MSC group (Fig. 3d) and resulted in an approximately threefold increase in the HGF secreted into the culture media while the opposite result was observed in the shHGF-MSC group (Fig. 3e). Therefore, all of the data suggested that the HGF gene of MSCs was overexpressed or knocked down at the mRNA and protein levels.

\section{Differentiation of mDCs into DCregs is attributed to MSCs secreting HGF}

To investigate whether the differentiation of $\mathrm{mDCs}$ into DCregs induced by MSCs is affected by HGF secretion, we again performed a DC phenotypic analysis to show that HGF-MSC-treated mDCs expressed fewer functional markers such as MHCII and CD86 than MSCDCs, but more than shHGF-MSC-treated mDCs (Fig. 4a). DCs were then independently cultured for another $24 \mathrm{~h}$ after thorough washes, and we observed that IL-10 and TGF- $\beta$ were increased in the MSC-DC and HGF-MSCDC groups, and IL-12 was decreased. After knocking down the HGF gene in MSCs, the regulatory effect of MSC-DCs on the above cytokines was weakened (Fig. 4b). In addition, compared with mDCs, the DCs cocultured with MSCs had stronger phagocytic capacity, and HGF-MSC-DCs had the strongest, followed by MSC-DCs and then shHGF-MSC-DCs (Fig. 4c). To investigate whether the ability of HGF-MSCs/shHGFMSC-DCs to stimulate lymphocyte proliferation was different from that of $\mathrm{mDCs}$ and MSC-DCs, a mitogen proliferative assay was performed. CFSE-labeled splenic lymphocytes were used as responders and cocultured with allogenic mDCs, MSC-DCs HGF-MSC-DCs, and shHGF-MSC-DCs. There was a gradient in lymphocyte proliferation according to the type of MSCs, with the greatest response observed in coculture with $\mathrm{mDCs}$, then shHGF-MSC-DCs and MSCs, and the least difference between groups for HGF-MSC-DCs (Fig. 4d, e). These results demonstrate that the HGF secreted by MSCs induces mDCs into immune-tolerant DCs, inhibits lymphocyte proliferation, and regulates the release of inflammatory cytokines. 
A
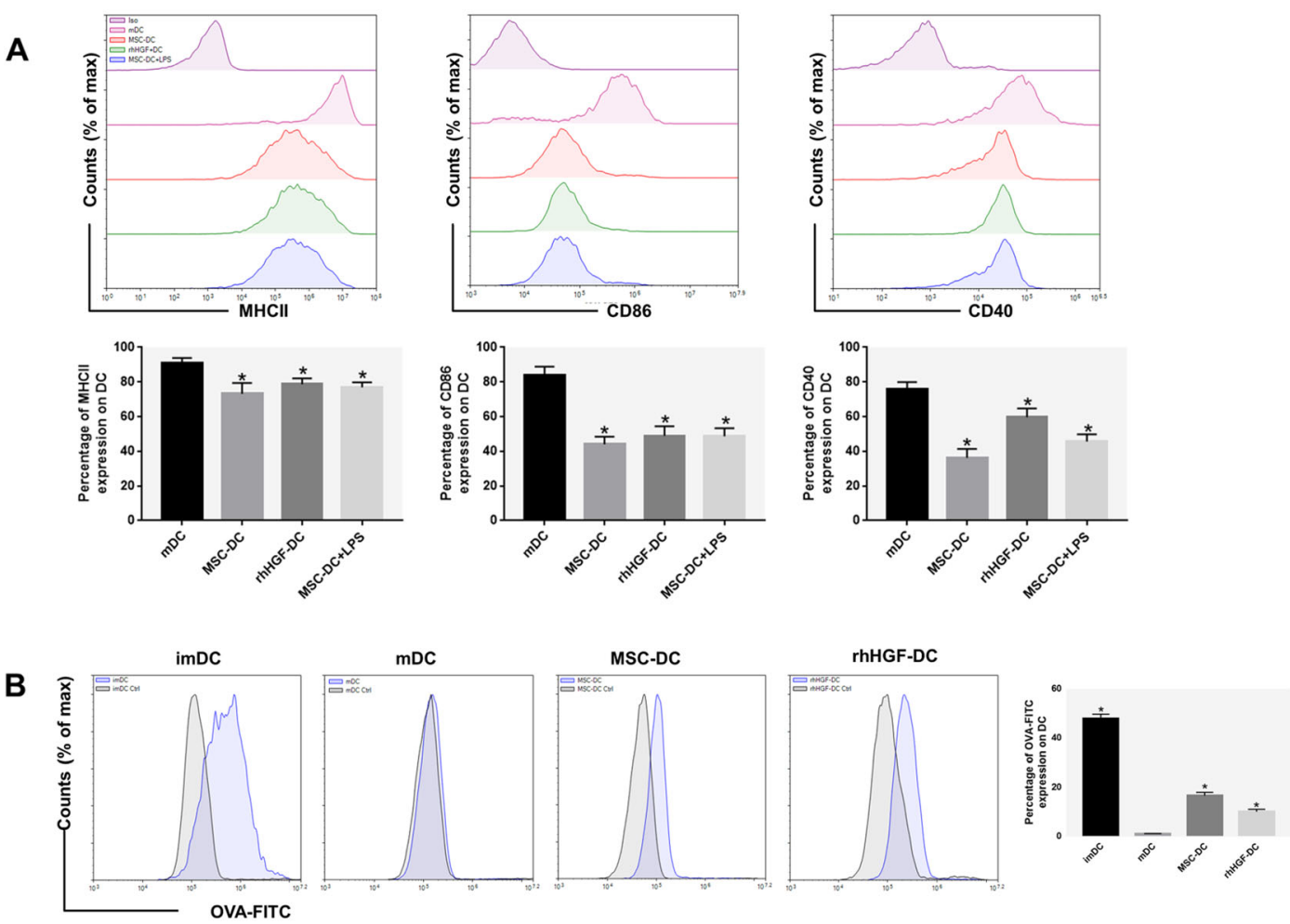

C
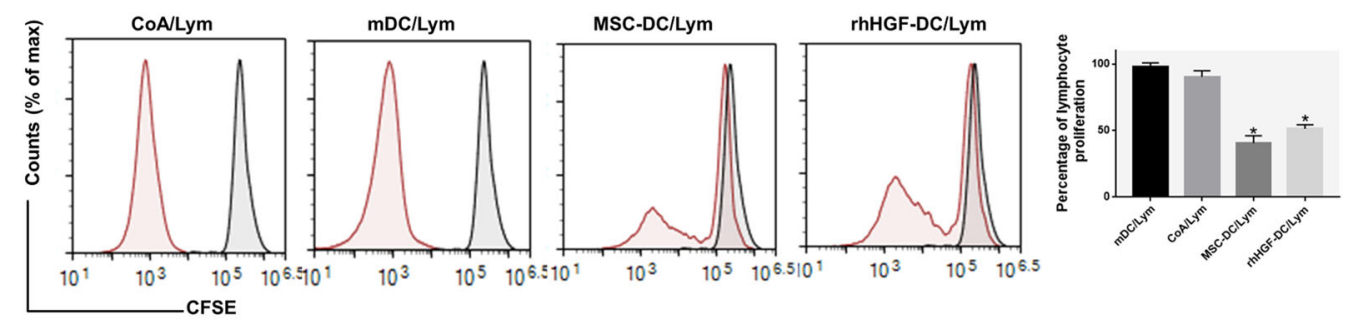

D
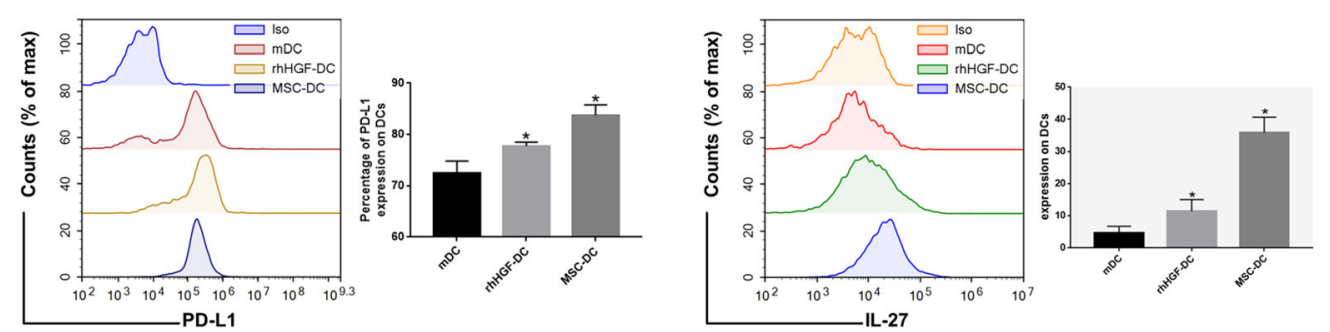

E
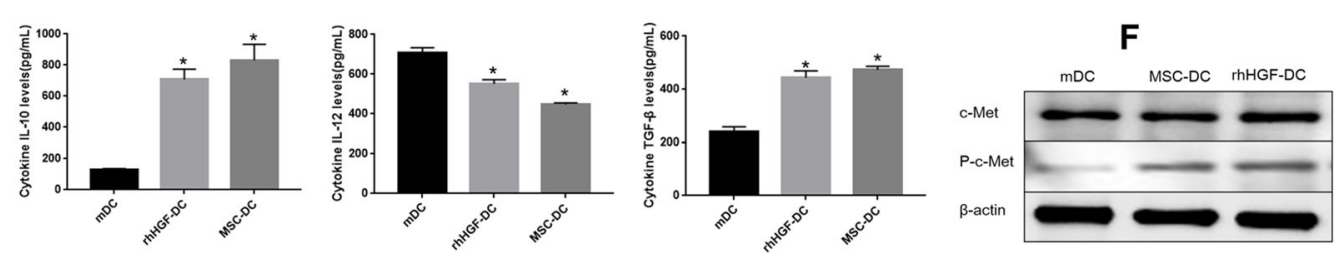

Fig. 2 (See legend on next page.) 
(See figure on previous page.)

Fig. 2 Effects of MSCs and rhHGF on mDC differentiation. a Immunophenotype analysis of DCs (expression of MHCll, CD86, and CD40 in mDCs cultured for $72 \mathrm{~h}$ in the presence of MSC or rhHGF, and expression of MHCII, CD86, and CD40 in MSC-induced DCs after LPS stimulation for $24 \mathrm{~h}$ ). Percentage of MHCll, CD86, and CD40 expression on DCs after $72 \mathrm{~h}$ of incubation with or without MSC or rhHGF. $\mathbf{b}$ Phagocytic ability analysis of DCs (expression of OVA-FITC in DCs and percentage of OVA-FITC-positive cells of DCs). c Lymphocyte proliferation stimulated by mitotic proenzyme ConA, mDCs, MSC-DCs, or rhHGF-DCs. Splenocytes from normal BALB/C mice were used as responder cells in the mitogen proliferative assay. mDCs, MSC-DCs, rhHGF-DCs, or ConA were used as stimulators. Splenocytes from normal mice served as controls. The proliferative responses were assessed by CFSE labeling and FACS (gray line, unstimulated spleen cell). $\mathbf{d}$ Immunophenotype analysis of DCs (expression of PD-L1 and IL-27 in mDCs cultured for $72 \mathrm{~h}$ in the presence of MSC or rhHGF). Percentage of PD-L1 and IL-27 expression in DCS after $72 \mathrm{~h}$ of incubation with or without MSC or rhHGF. e Cytokine secretion profiles of mDCs, MSC-DCs, and rhHGF-DCs after culture for $24 \mathrm{~h}$. $\mathbf{f}$ Changes in c-met and phosphorylated c-met levels in dendritic cells cultured for $72 \mathrm{~h}$ in the presence of MSCs or rhHGF ( $n=3,{ }^{*} P<0.05$ versus $\mathrm{mDC}$ group; data are expressed as mean $\pm \mathrm{SD}$ ). Each experiment was repeated times

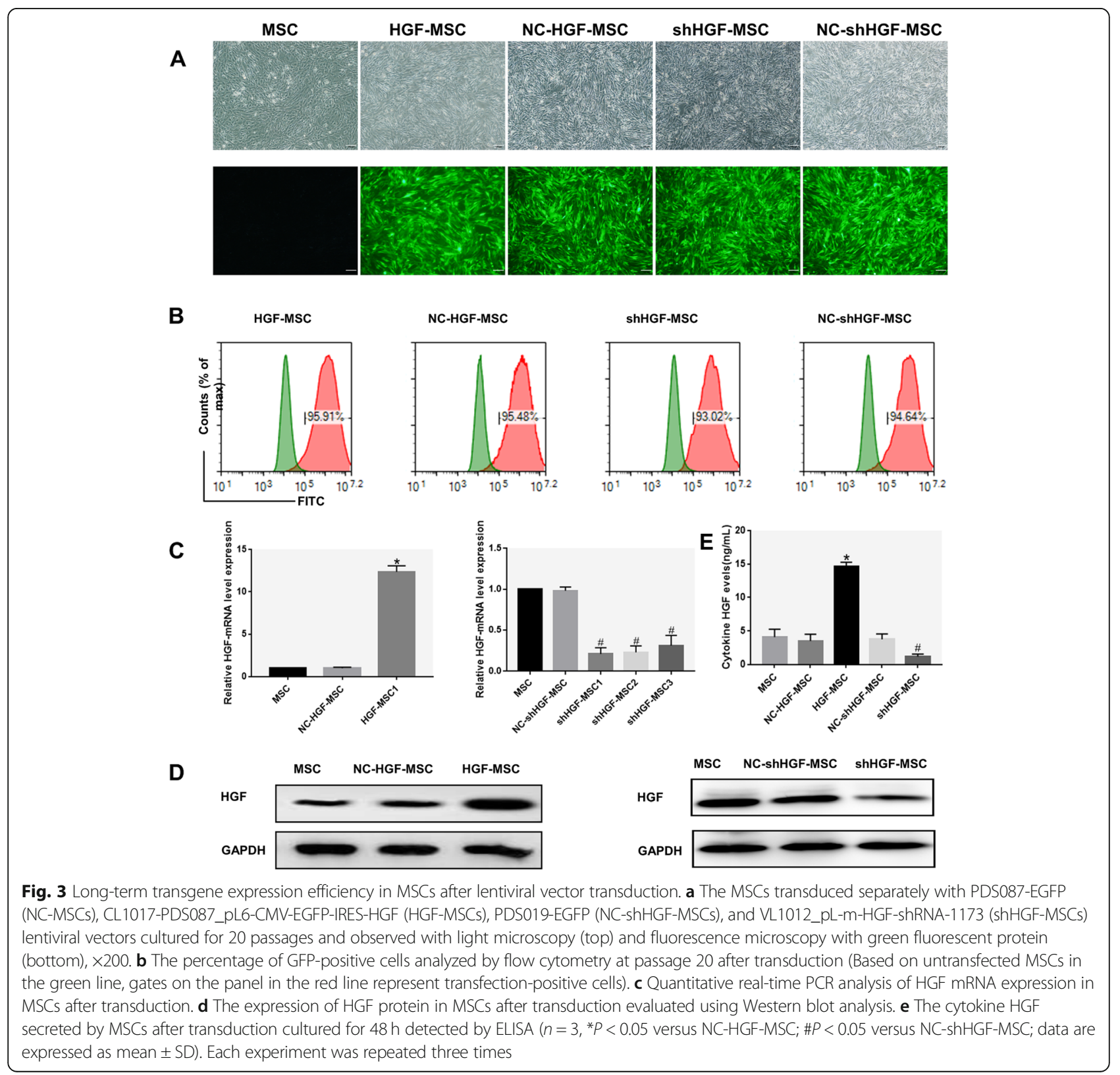




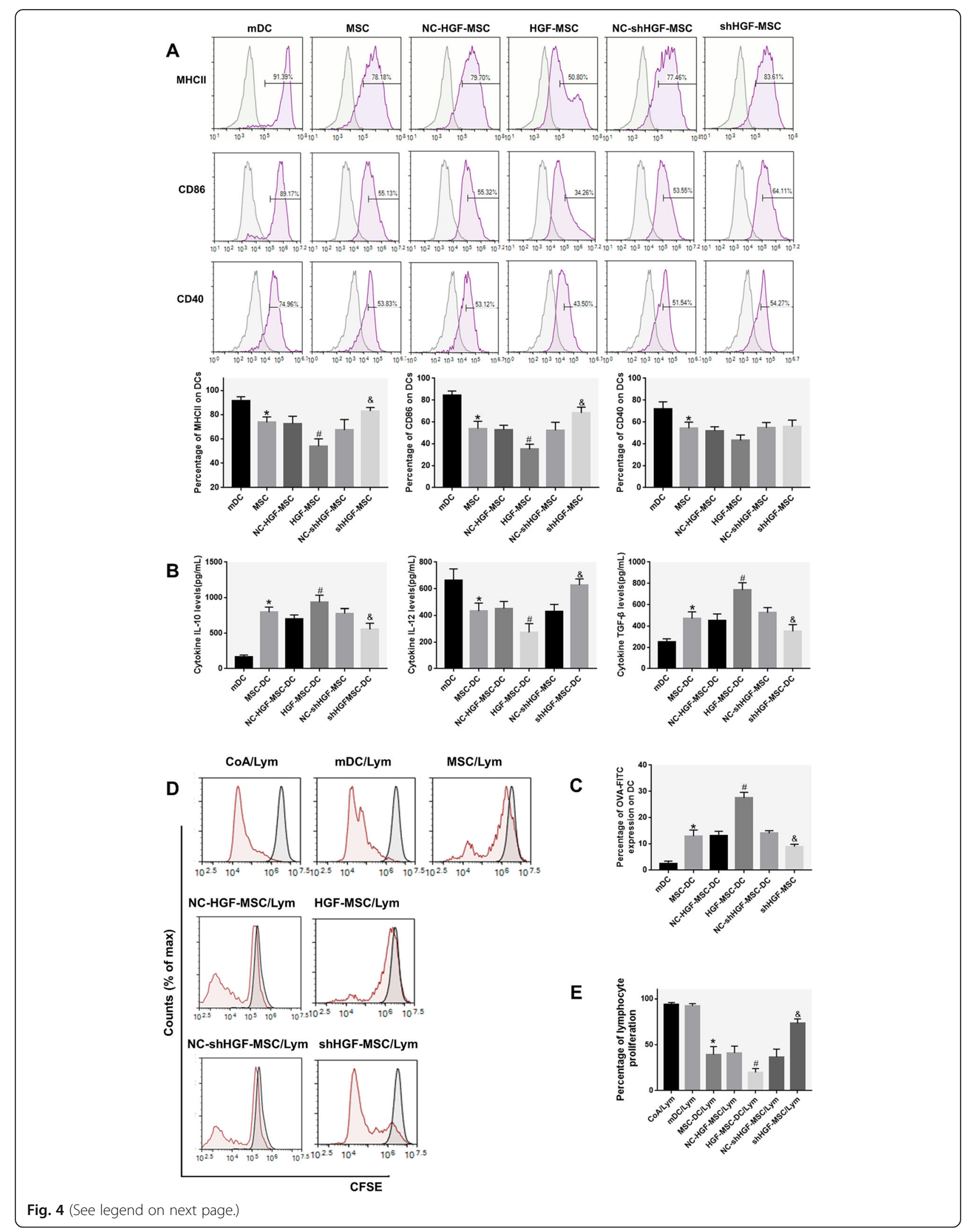


(See figure on previous page.)

Fig. 4 HGF plays a role in the MSC-induced differentiation of DCs. a Immunophenotype analysis of DCs (expression of MHCII, CD86, and CD40 on $\mathrm{mDCs}$ cultured for $72 \mathrm{~h}$ in the presence of MSCs or MSCs after transduction). The percentage of DCs positive for MHCII, CD86, and CD40 after coculture for $72 \mathrm{~h}$ with MSCs or transduced MSCs. b Cytokine secretion profiles of mDCs, MSC-DCs, NC-HGF-MSC-DCs, HGF-MSC-DCs, NC-shHGFMSC-DCs, and shHGF-MSC-DCs after culture for $24 \mathrm{~h}$. c Phagocytic ability analysis of MSC-induced DCs (percentage of OVA-FITC-positive cells). $\mathbf{d}$, e Lymphocyte proliferation stimulated by ConA, mDCs, MSC-DCs, NC-HGF-MSC-DCs, HGF-MSC-DCs, NC-shHGF-MSC-DCs, and shHGF-MSC-DCs. Normal BALB/C mouse solenocytes were used as responder cells in the mitogen proliferative assay. The proliferative responses were assessed by CFSE labeling and FACS. (Inside the gray line, there are unstimulated splenic cells.) ( $n=3,{ }^{*} P<0.05$ versus mDC; $\# P<0.05$ versuS NC-HGF-MSC; $\& P<0.05$ versus NC-shHGF-MSC; data are expressed as mean \pm SD). Each experiment was repeated at least three times

\section{HGF induces the differentiation of $\mathrm{mDCs}$ into DCregs via the AKT signaling pathway}

To directly examine the mechanism of Akt in DCreg generation, we tested the phosphorylation level of the Akt protein by Western blot. Surprisingly, we observed that mDCs cocultured with MSCs had more p-Akt molecules than mDCs. When mDCs were cocultured with HGF-MSCs, Akt phosphorylation increased, while it decreased when $\mathrm{mDCs}$ were cocultured with shHGF-MSC (Fig. 5a). Collectively, these data show that the HGF effects are associated with the stimulation of Akt signaling during the differentiation of $\mathrm{mDCs}$ into DCregs.

Furthermore, we added AKT inhibitor (MK-2206) in the rhHGF and DC culture system and observed that the immunomodulatory effects of rhHGF were significantly attenuated in the Akt inhibitor group. Higher levels of MHCII, CD86, and CD40 were expressed in the Akt inhibitor group compared with the rhHGF-DC group (Fig. 5b, c). HGF reduced the secretion of IL-12 and increased the secretion of IL-10 and TGF- $\beta$ in DCs, and these immunomodulatory effects were inhibited by MK-2206 (Fig. 5d). Inhibition of Akt increased the DC phagocytosis and lymphocyte proliferation stimulated by treatment with HGF (Fig. 5e, f). These results indicate that MK-2206 weakens the regulatory effect of HGF and that Akt activation is involved in the immune regulation of DCs.

\section{The tolerogenic DCs induced by MSC-secreted HGF attenuate $\mathrm{ALI}$}

To verify the regulation of MSCs on lung DCs in ALI mice and determine if its mechanism is related to the secretion of HGF, we treated lung DCs with MSCs, HGF-MSCs, and shHGF-MSCs $6 \mathrm{~h}$ after ALI model establishment and compared their effects on mDCs. In unchallenged mice, the frequency of $\mathrm{CD} 11 \mathrm{c}^{+} \mathrm{CD} 11 \mathrm{~b}^{+}$ $\mathrm{cDCs}$ in the lung tissue was low, while the frequency of lung cDCs was significantly increased $24 \mathrm{~h}$ after airway injection of LPS and was reduced after treatment with MSC. Treatment of ALI mice with HGF-MSCs further reduced DC aggregation, while treatment with shHGFMSCs increased lung DC aggregation (Fig. 6a). These results demonstrate a rapid increase in total lung $\mathrm{CDC}$ recruitment in LPS-induced ALI mice and show that MSCs inhibit lung DC aggregation by secreting HGF.
MDCs are characterized by a further increase in the expression of the stimulatory molecules CD40, CD80, CD86, and MHCII on their surface. We analyzed the maturation status of lung cDCs based on the expression of CD86 and MHCII. At baseline, lung CDCs in the control group at $24 \mathrm{~h}$ expressed relatively low levels of CD86 and MHCII (Fig. 6b, c). In parallel to the accumulation of pulmonary cDCs in ALI mice, the expression of CD86 and MHCII on the surface of respiratory cDCs showed a significant increase. Notably, treatment with MSCs led to a marked reduction in CD86 or MHCII expression on pulmonary cDCs. Treatment with HGFMSCs resulted in a significant reduction in CD86 on lung $\mathrm{CDC}$ compared to treatment with the empty vector control group but increased after treatment with shHGF-MSCs (Fig. 6c). Then, we used a histological evaluation of the lungs to confirm the effect of different MSC treatments on LPS-induced lung injury in mice (Fig. 6d). Lung specimens from ALI mice showed extensive 24-h alveolar wall thickness caused by edema, as well as significant inflammatory cell infiltration that were improved after MSC treatment. High expression of the HGF gene promoted MSCs to alleviate lung pathological damage, and knockdown of the HGF gene expression inhibited the effect of the MSC treatment (Fig. 6d). The LWW/BW response to pulmonary edema is related to the severity of lung injury [35]. The LWW/ BW in ALI mice was significantly higher than that in control mice at $24 \mathrm{~h}$, indicating that LPS administration effectively induced ALI (Fig. 6e). In contrast, when ALI mice were treated with MSC, the LWW/BW was significantly lower than that of ALI. Treatment with HGFMSC resulted in a significant reduction in LWW/BW compared to the empty vector control group but increased after treatment with shHGF-MSCs (Fig. 6e). These results indicated that MSCs are dependent on HGF to attenuate LPS-induced ALI.

\section{Discussion}

Here, we uncover that MSCs alleviate ALI via paracrine HGF regulation of DC immune function. Our findings suggest that imDCs are induced to mature after $24 \mathrm{~h}$ of LPS $(50 \mathrm{ng} / \mathrm{ml})$ stimulation. Via the HGF/Akt pathway, MSCs induced mDCs to differentiate into DCregs that 


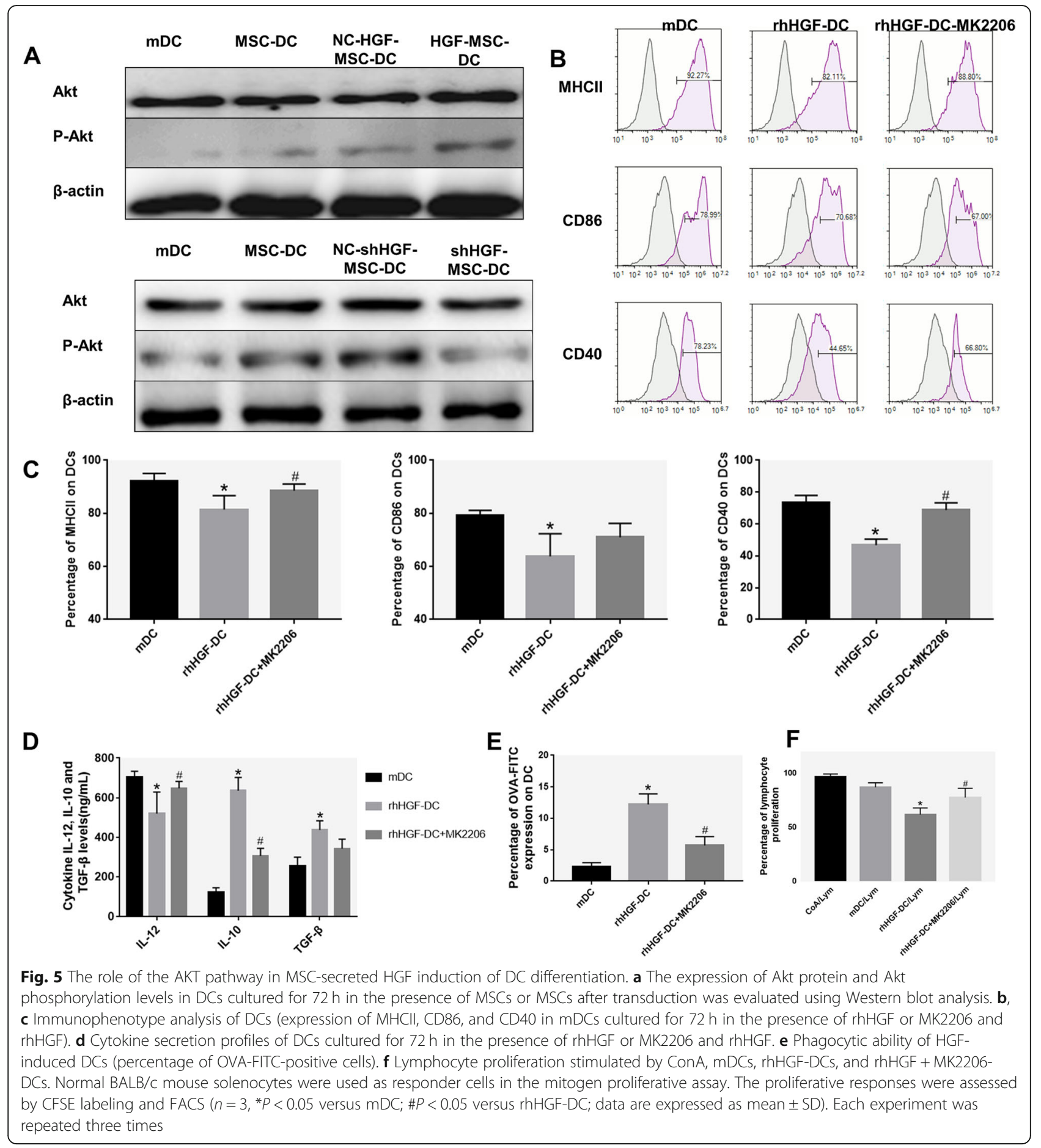

have low expression of MHCII, CD86, and CD40 molecules, strong phagocytic function, and weakened promotion of $\mathrm{T}$ cells. MSCs decreased lung DC aggregation and maturity and alleviated lung pathological injury in LPS-induced ALI mice. High expression of the HGF gene enhanced the above effects of MSCs, while decreased expression of HGF weakened the above effects of MSCs.
The induction of mDCs into DCregs is more likely to be the key to early ALI treatment. DCs are present throughout the lung tissue, underlying the epithelial layer, and under steady-state conditions have fewer numbers and immature phenotypes and are ready to encounter foreign material, infection, or tissue damage $[16,36]$. After inhalation of bacterial stimulation, airway mucosal DCs accelerate antigen sampling and transport, 
A
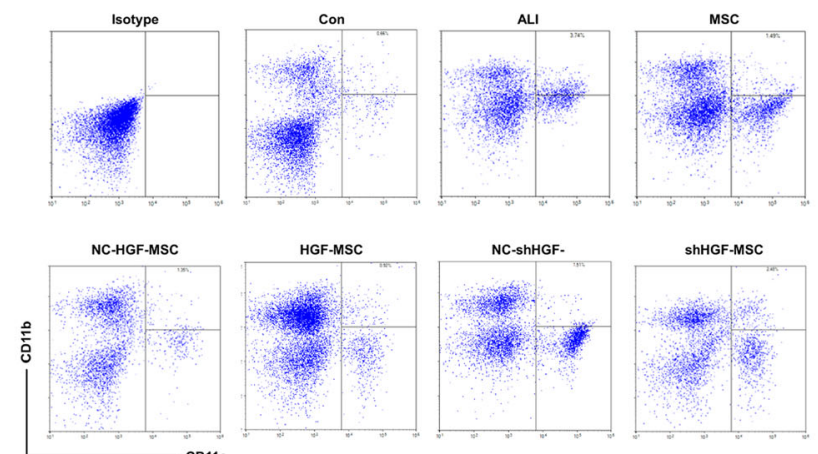

B
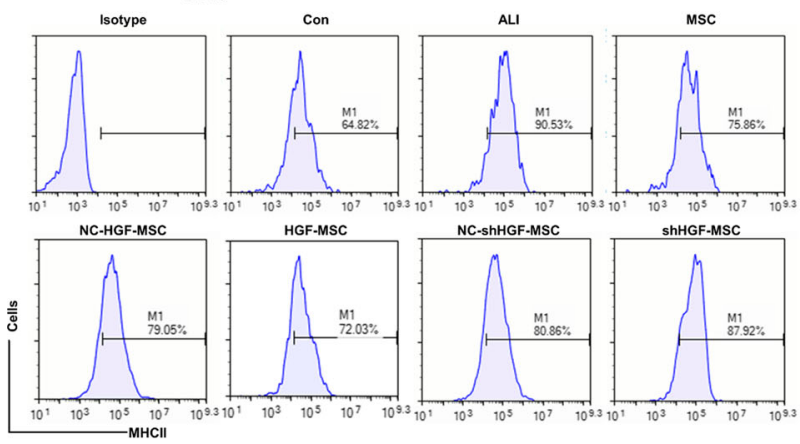

C
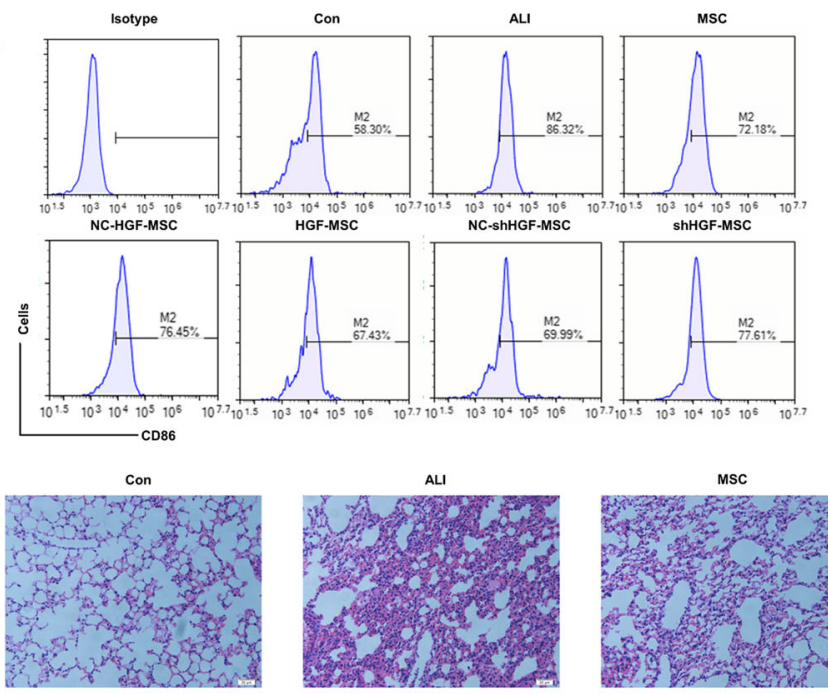

NC-HGF-MSC

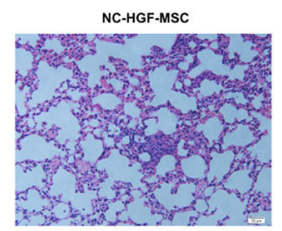

NC-shHGF-MSC

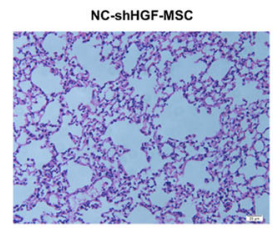

Msc
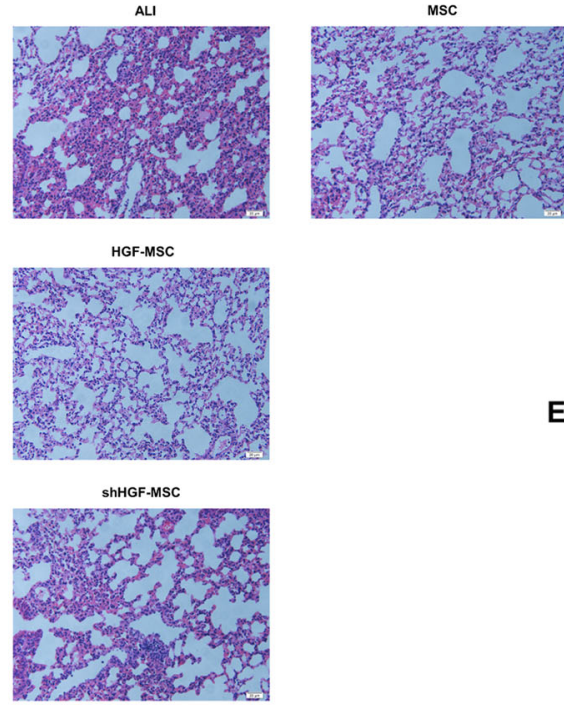
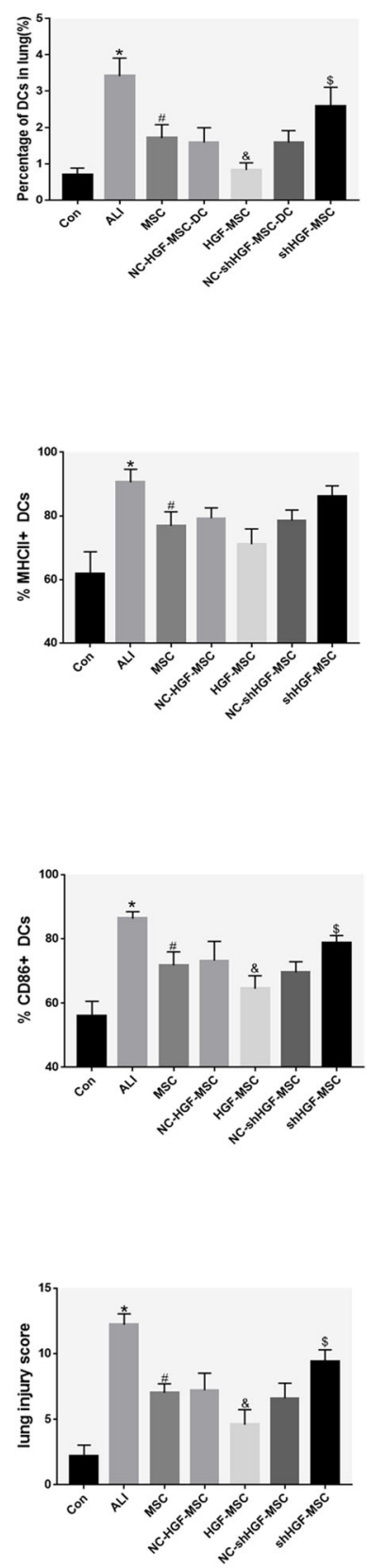

E

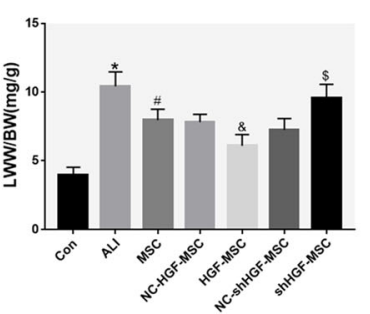

Fig. 6 (See legend on next page.) 


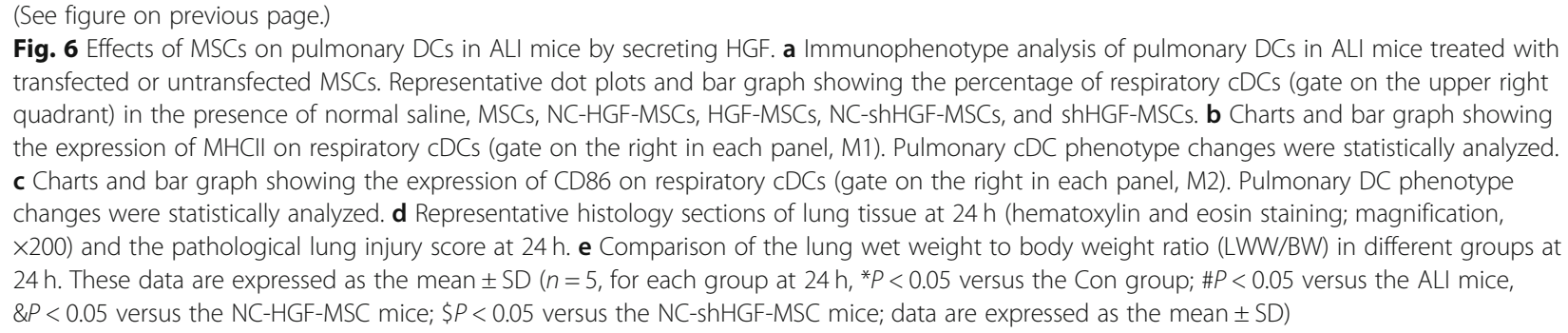

reaching regional lymph nodes and expressing complete APC activity within $30 \mathrm{~min}$ after microbial exposure [37]. DCs rapidly accumulate in the lungs, reaching a peak within $2 \mathrm{~h}$ and maturation within $6 \mathrm{~h}$ after the inhalation of pathogenic substances [14, 38]. Our study found that the accumulation of DCs in mouse lungs remained significant $24 \mathrm{~h}$ after inducing ALI, and the in vitro experiments also showed that the phagocytosis of DCs after LPS stimulation was significantly reduced. Once mobilized into the lungs, the DCs sample the incoming antigen and undergo a maturation process characterized by the upregulation of cell surface expression of MHCII and costimulatory molecules (e.g., CD80 and CD86) [14]. Similar phenotypes were detected in the lungs of ALI mice in this study. ARDS is an ALI mediated by an uncontrolled inflammatory response [39], and mDCs play an important immunomodulatory role during the ARDS exacerbation process $[16,36]$. Therefore, regulating the differentiation of $\mathrm{mDCs}$ into DCregs may improve ALI.

Studies have shown that mDCs are induced by MSCs to differentiate into DCregs with reduced expression of MHCII, CD11c, CD80, CD86, and CD40 [18, 19], but whether this phenomenon regulates the immune response in ARDS is unclear. The results showed that MSCs may induce mDCs into a novel DCreg population with high endocytosis and downregulated expression of the costimulatory molecules MHCII, CD86, and CD40. LPS stimulation did not reverse this trend, demonstrating that a different DC population was induced by MSCs. This is consistent with previous reports [19]. Our further in vivo study showed that the number of conventional DCs and mDCs in the lung decreased and lung injury and pulmonary water content were reduced in the MSC-treated group compared with the ALI group. Our data demonstrated that MSCs induces the transformation of mDCs into DCregs to reduce ALI. However, the mechanism by which MSCs regulate DCs to treat early ALI remains unclear.

Paracrine HGF is an important mechanism by which MSCs regulates DCs to alleviate ARDS lung injury. Since the discovery and characterization of the epithelial specific growth factors keratinocyte growth factor (KGF) and HGF, their role in lung development, lung inflammation, and repair has been extensively studied $[40,41]$. Our previous research also showed that the ability to express HGF was required for MSCs to protect the injured lung [26]. However, an RCT by McAuley DF et al. found that KGF was not beneficial for physiological outcomes in ARDS and could make clinical outcomes worse [41], which was quite different from the results of animal experiments [42 43]. The reasons may be as follows: the effect of giving KGF before lung injury is better than that after injury, local administration of intratracheal is better than systemic [42], heterogeneity for different reasons makes ARDS patients to have different effects on treatment, or exogenous KGF supplementation is not related to any other beneficial effects. In addition, unlike KGF, others and our studies have shown that in addition to repairing epithelial damage, HGF not only has the effect of repairing epithelial damage but also repairs endothelial damage and immune regulation $[26,29,43,45]$. Consistent with previous reports, HGF increased the expression of PD-L1 on DCs that mediate regulatory $\mathrm{T}$ cell induction and tolerance $[28,44]$. Our results also indicate that MSC or HGF treatment significantly increased PD-L1 expression in DCs. Therefore, HGF is more likely to be an ARDS treatment option than KGF. HGF produced by MSCs also mediates T cell suppression [45] and is involved in the inhibition of imDC activation [46]. Studying the process of MSCinduced DC immune tolerance, we also unexpectedly found that the phosphorylation level of the HGF receptor c-Met protein in DCs was significantly increased, so we speculated that HGF/c-met may be an important link in the MSC-mediated DC production. Further verification results showed that HGF could induce mDCs to produce an immunotolerant phenotype, such as MSCDC, and HGF-DCs can also reduce lymphocyte proliferation and IL- 12 secretion and increase TGF- $\beta$ and IL-10 production compared with mDCs. These results revealed that MSC-induced DC immune tolerance may be related to HGF secretion.

That MSCs alleviate lung injury by secreting HGF has been confirmed in vivo and in vitro [26, 47], but whether it is related to DCreg generation is still unknown. Compared with NC-HGF-MSC-DCs, DCs obtained by coculture with the HGF overexpressing HGF-MSCs had 
lower levels of mDC markers, IL-12 secretion, and ability to induce the proliferation of lymphocytes after stimulation, but TGF- $\beta$ and IL-10 increased more significantly. In contrast, the immunosuppressive effects of MSCs on $\mathrm{mDCs}$ were downregulated in the shHGF-MSC group. Thus, shHGF-MSC-DCs expressed higher levels of mature functional markers, secreted higher levels of IL-12, and induced the proliferation of more lymphocytes after coculture and had a larger decrease in TGF- $\beta$ and IL- 10 . The secretion of inflammatory cytokines was consistent with previous studies. MSCs inhibited the secretion of the pro-inflammatory factor IL-12 [48], promoting the secretion of the anti-inflammatory factor TGF- $\beta$ and IL$10[26,49]$. As expected, in animal studies, compared with the MSC treatment group, treatment MSCs with an upregulated HGF secretion led to improvement of acute lung inflammation and lung injury and promoted mDCs into DCregs in the murine model of LPS-induced ALI, but the opposite results were observed in the shHGF-MSC treatment group. The results indicated that MSCs were partially dependent on the secretion of HGF to induce $\mathrm{mDC}$ immune tolerance for the treatment of early ALI, but the downstream mechanism of HGF was still unclear.

Previous studies have shown that HGF can inhibit the activation of conventional DCs via the c-Src-PI3K-AKTmTOR-GSK3 $\beta$ pathway $[46,50]$. Singhal et al. found that proximal signal transduction events induced in dendritic cells by HGF include the physical association of c-Src with the HGF receptor c-MET and the associated activation of c-Src. Activation of c-Src, in turn, establishes a complex of phosphatidylinositol 3-kinase and c-MET and promotes downstream activation of phosphatidylinositol 3-kinase/ AKT (Ser473). Notably, activation of c-Src stimulated by HGF leads to induction of phosphatidylinositol 3-kinase complexes $\mathrm{p} 85 \alpha / \mathrm{p} 110 \alpha$ and $\mathrm{p} 85 \alpha / \mathrm{p} 110 \delta$, which are necessary to activate the target of rapamycin [46]. This study found similar results that the phosphorylation levels of cMet and Akt (Ser473) were significantly increased in MSC-treated DCs. Therefore, this result suggests that the c-Met/Akt pathway is involved in the HGF mechanism of inducing $\mathrm{mDC}$ immune tolerance production. The phosphorylation of c-Met and Akt in DCs was positively correlated with HGF expression in MSCs, indicating that c-Met/Akt is located downstream of HGF during the induction of DCreg production. In this study, the expression of costimulatory molecules in DCs and functional markers of T cells stimulated by DCs showed that HGF could induce DCreg production, and Akt inhibitors could reverse the result. Consequently, these results revealed that the disruption of Akt phosphorylation at Ser473 downregulated the effect of $\mathrm{HGF}$ on DC regulation. Thus, these data suggest that MSCs might activate the HGF/Akt pathway, promoting the differentiation of $\mathrm{mDCs}$ into DCregs to reduce ALI, but the mechanism downstream of AKT remains to be elucidated in future experiments. There has been a lot of progress in the knowledge of the upstream regulatory inputs into AKT, but key multi-function downstream signaling nodes (GSK3, FoxO, mTORC1) greatly expand the functional range of AKT [51]. According to previous studies [46], HGF treatment caused sequential activation cascade $\mathrm{c}-\mathrm{Src} \rightarrow \mathrm{PI} 3 \mathrm{~K} \rightarrow \mathrm{AKT} \rightarrow \mathrm{mTOR}$ in DCs, and activation of mTOR through the pathway c-SrcPI3K-AKT depends on HGF-stimulated GSK3 beta inactivation. This may be an important mechanism by which MSC secretes HGF-induced DC to alleviate ALI.

\section{Conclusions}

This study showed that the induction of mDC immune tolerance is an important process for MSCs to alleviate early ALI, and this process is closely related to paracrine HGF. These findings provide new insights into the role of MSCs in the secretion of HGF to repair lung injury. We believe that these studies will enhance our understanding of ARDS/ALI immunotherapy.

\section{Abbreviations \\ Abs: Antibodies; ALI: Acute lung injury; ANOVA: Analysis of variance; ARDS: Acute respiratory distress syndrome; BM: Bone marrow; BSA: Bovine serum albumin; CDCs: Conventional DCs; CFSE: Carboxyfluorescein diacetate succinimidyl ester; ConA: Concanavalin A; DCregs: Regulatory DCs; DCs: Dendritic cells; ECL: Enhanced chemiluminescence; EGFP: Enhanced green fluorescent protein; ELISA: Enzyme-linked immunosorbent assay; FBS: Fetal bovine serum; GM-CSF: Granulocyte-macrophage colony- stimulating factor; HGF: Hepatocyte growth factor; IL-10: Interleukin-10; IL- 12: Interleukin-12; IL-4: Interleukin-4; imDCs: Immature DCs; \\ LPS: Lipopolysaccharide; LWW/BW: Lung wet weight to body weight ratio; mDCs: Mature DCs; MSC: Mesenchymal stem cell; PBS: Phosphate-buffered saline; PD-L1: Programmed death ligand 1; PVDF: Polyvinylidene difluoride; SD: Standard deviation; TGF- $\beta$ : Transforming growth factor beta}

\section{Acknowledgements}

We would like to thank Dr. Feng Liu and Fei Peng for their technical support on HGF transduction and coculture of mesenchymal stem cells with DCs and Dr. Cuilin Chu for her advice and guidance on writing.

\section{Authors' contributions}

Z-HL and WC participated in the collection and assembly of data, data analysis and interpretation, and manuscript writing. S-SM and X-PX participated in the acquisition, analysis, and interpretation of data. J-FX participated in the collection and assembly of the data and review of the manuscript. F-MG, YY and $\mathrm{H}-\mathrm{BQ}$ participated in the design and supervision of research. $\mathrm{LL}$ conceived of the study, performed the statistical analysis, and helped to draft the manuscript. All authors read and approved the final manuscript.

\section{Funding \\ Contract grant sponsor: National Natural Science Foundation of China; contract grant numbers: 81670074, 81000822, 81801897, and 81871602 Contract grant sponsor: Social Development Project or National Natural Science Foundation of Jiangsu Province; contract grant number: BE2018743, BK20171271. Contract grant sponsor: Jiangsu Province Key Medical Discipline; contract grant number: ZDXKA2016025.}

\section{Availability of data and materials}

All datasets used and/or analyzed during the current study are available from the corresponding author on reasonable request.

Ethics approval and consent to participate

All of the experimental procedures were approved by the Southeast University Ethics Committee (protocol number 20170105007). 


\section{Consent for publication}

Not applicable.

\section{Competing interests}

The authors declare that they have no competing interests.

Received: 30 July 2019 Revised: 28 October 2019

Accepted: 5 November 2019 Published online: 04 December 2019

\section{References}

1. Kido T, Muramatsu K, Asakawa T, Otsubo H, Ogoshi T, Oda K, et al. The relationship between high-dose corticosteroid treatment and mortality in acute respiratory distress syndrome: a retrospective and observational study using a nationwide administrative database in Japan. BMC Pulm Med. 2018; 18:28.

2. Potapov AL, Novikov N, Tumanskii VA, Babanin AA. Surfactant replacement therapy increases life-span of patients with acute respiratory distress syndrome. Klin Khir. 2013;2:57-9.

3. Gebistorf F, Karam O, Wetterslev J, Afshari A. Inhaled nitric oxide for acute respiratory distress syndrome (ARDS) in children and adults. Cochrane Database Syst Rev. 2016;2016:Cd002787.

4. Al-Timemy AH, Khushaba RN, Escudero J. Selecting the optimal movement subset with different pattern recognition based EMG control algorithms. Conf Proc IEEE Eng Med Biol Soc. 2016;2016:315-8.

5. Lawler PR, Fan E. Heterogeneity and phenotypic stratification in acute respiratory distress syndrome. Lancet Respir Med. 2018:6:651-3.

6. Samary CS, Moraes L, Santos CL, Huhle R, Santos RS, Ornellas DS, et al. Lung functional and biologic responses to variable ventilation in experimental pulmonary and extrapulmonary acute respiratory distress syndrome. Crit Care Med. 2016:44:e553-62.

7. Lin S, Wu H, Wang C, Xiao Z, Xu F. Regulatory T cells and acute lung injury: cytokines, uncontrolled inflammation, and therapeutic implications. Front Immunol. 2018:9:1545.

8. Thompson BT, Chambers RC, Liu KD. Acute respiratory distress syndrome. N Engl J Med. 2017;377:562-72.

9. Fan E, Brodie D, Slutsky AS. Acute respiratory distress syndrome: advances in diagnosis and treatment. JAMA. 2018:319:698-710.

10. Matthay MA, Zemans RL. The acute respiratory distress syndrome: pathogenesis and treatment. Annu Rev Pathol. 2011;6:147-63.

11. Kopf M, Schneider C, Nobs SP. The development and function of lungresident macrophages and dendritic cells. Nat Immunol. 2015;16:36-44.

12. Yi S, Zhai J, Niu R, Zhu G, Wang M, Liu J, et al. Eosinophil recruitment is dynamically regulated by interplay among lung dendritic cell subsets after allergen challenge. Nat Commun. 2018:9:3879.

13. Buttignol M, Pires-Neto RC, Rossi ESRC, Albino MB, Dolhnikoff M, Mauad T. Airway and parenchyma immune cells in influenza a(H1N1)pdm09 viral and non-viral diffuse alveolar damage. Respir Res. 2017;18:147.

14. Liu J, Zhang PS, Yu Q, Liu L, Yang Y, Guo FM, et al. Losartan inhibits conventional dendritic cell maturation and Th1 and Th17 polarization responses: novel mechanisms of preventive effects on lipopolysaccharideinduced acute lung injury. Int J Mol Med. 2012;29:269-76.

15. Bantsimba-Malanda C, Marchal-Somme J, Goven D, Freynet O, Michel L, Crestani B, et al. A role for dendritic cells in bleomycin-induced pulmonary fibrosis in mice? Am J Respir Crit Care Med. 2010;182:385-95.

16. Dong L, He HL, Lu XM, Yang Y, Qiu HB. Modulation of FLT3 signaling targets conventional dendritic cells to attenuate acute lung injury. Apmis. 2012;120:808-18.

17. Liu Y, Yin Z, Zhang R, Yan K, Chen L, Chen F, et al. MSCs inhibit bone marrow-derived DC maturation and function through the release of TSG-6. Biochem Biophys Res Commun. 2014;450:1409-15.

18. Djouad F, Charbonnier LM, Bouffi C, Louis-Plence P, Bony C, Apparailly $F$, et al. Mesenchymal stem cells inhibit the differentiation of dendritic cells through an interleukin-6-dependent mechanism. Stem Cells. 2007; 25:2025-32

19. Zhang B, Liu R, Shi D, Liu X, Chen Y, Dou X, et al. Mesenchymal stem cells induce mature dendritic cells into a novel Jagged-2-dependent regulatory dendritic cell population. Blood. 2009;113:46-57.

20. Deng Y, Yi S, Wang G, Cheng J, Zhang Y, Chen W, et al. Umbilical cordderived mesenchymal stem cells instruct dendritic cells to acquire tolerogenic phenotypes through the IL-6-mediated upregulation of SOCS1. Stem Cells Dev. 2014;23:2080-92.
21. Jiang XX, Zhang Y, Liu B, Zhang SX, Wu Y, Yu XD, et al. Human mesenchymal stem cells inhibit differentiation and function of monocytederived dendritic cells. Blood. 2005;105:4120-6.

22. Jackson MV, Morrison TJ, Doherty DF, Mcauley DF, Matthay MA, Kissenpfennig A, et al. Mitochondrial transfer via tunneling nanotubes is an important mechanism by which mesenchymal stem cells enhance macrophage phagocytosis in the in vitro and in vivo models of ARDS. Stem Cells. 2016;34:2210-23.

23. Lee FY, Chen KH, Wallace CG, Sung PH, Sheu JJ, Chung SY, et al. Xenogeneic human umbilical cord-derived mesenchymal stem cells reduce mortality in rats with acute respiratory distress syndrome complicated by sepsis. Oncotarget. 2017:8:45626-42.

24. Liu L, He H, Liu A, Xu J, Han J, Chen Q, et al. Therapeutic effects of bone marrow-derived mesenchymal stem cells in models of pulmonary and extrapulmonary acute lung injury. Cell Transplant. 2015;24:2629-42.

25. He H, Liu L, Chen Q, Liu A, Cai S, Yang Y, et al. Mesenchymal stem cells overexpressing angiotensin-converting enzyme 2 rescue lipopolysaccharideinduced lung injury. Cell Transplant. 2015;24:1699-715.

26. Hu S, Li J, Xu X, Liu A, He H, Xu J, et al. The hepatocyte growth factorexpressing character is required for mesenchymal stem cells to protect the lung injured by lipopolysaccharide in vivo. Stem Cell Res Ther. 2016;7:66.

27. Yang Y, Chen QH, Liu AR, Xu XP, Han JB, Qiu HB. Synergism of MSCsecreted HGF and VEGF in stabilising endothelial barrier function upon lipopolysaccharide stimulation via the Rac1 pathway. Stem Cell Res Ther. 2015;6:250.

28. Benkhoucha M, Molnarfi N, Dunand-Sauthier I, Merkler D, Schneiter G, Bruscoli S, et al. Hepatocyte growth factor limits autoimmune neuroinflammation via glucocorticoid-induced leucine zipper expression in dendritic cells. J Immunol. 2014;193:2743-52.

29. Baek JH, Birchmeier C, Zenke M, Hieronymus T. The HGF receptor/met tyrosine kinase is a key regulator of dendritic cell migration in skin immunity. J Immunol. 2012;189:1699-707.

30. Okunishi K, Dohi M, Nakagome K, Tanaka R, Mizuno S, Matsumoto K, et al. A novel role of hepatocyte growth factor as an immune regulator through suppressing dendritic cell function. J Immunol. 2005;175:4745-53.

31. Benkhoucha M, Santiago-Raber ML, Schneiter G, Chofflon M, Funakoshi H, Nakamura T, et al. Hepatocyte growth factor inhibits CNS autoimmunity by inducing tolerogenic dendritic cells and CD25+Foxp3+ regulatory T cells. Proc Natl Acad Sci U S A. 2010;107:6424-9.

32. Lutz MB, Kukutsch N, Ogilvie AL, Rossner S, Koch F, Romani N, et al. An advanced culture method for generating large quantities of highly pure dendritic cells from mouse bone marrow. J Immunol Methods. 1999; 223:77-92.

33. Cai SX, Liu AR, Chen $\mathrm{S}, \mathrm{He} H \mathrm{~L}$, Chen $\mathrm{QH}, \mathrm{Xu} J \mathrm{Y}$, et al. Activation of Wnt/ beta-catenin signalling promotes mesenchymal stem cells to repair injured alveolar epithelium induced by lipopolysaccharide in mice. Stem Cell Res Ther. 2015;6:65.

34. Chen QH, Liu AR, Qiu HB, Yang Y. Interaction between mesenchymal stem cells and endothelial cells restores endothelial permeability via paracrine hepatocyte growth factor in vitro. Stem Cell Res Ther. 2015;6:44.

35. Chen $\mathrm{TH}$, Wang JJ. Niacin pretreatment attenuates ischemia and reperfusion of pancreas-induced acute pancreatitis and remote lung injury through suppressing oxidative stress and inflammation and activation of SIRT1. Transplant Proc. 2018;50:2860-3.

36. Cook PC, Macdonald AS. Dendritic cells in lung immunopathology. Semin Immunopathol. 2016;38:449-60.

37. Jahnsen FL, Strickland DH, Thomas JA, Tobagus IT, Napoli S, Zosky GR, et al. Accelerated antigen sampling and transport by airway mucosal dendritic cells following inhalation of a bacterial stimulus. J Immunol. 2006;177:5861-7.

38. Vermaelen K, Pauwels R. Pulmonary dendritic cells. Am J Respir Crit Care Med. 2005;172:530-51.

39. Howell MD, Davis AM. Management of ARDS in adults. Jama. 2018;319: $711-2$.

40. Ware LB, Matthay MA. Keratinocyte and hepatocyte growth factors in the lung: roles in lung development, inflammation, and repair. Am J Physiol Lung Cell Mol Physiol. 2002;282:L924-40.

41. Lindsay CD. Novel therapeutic strategies for acute lung injury induced by lung damaging agents: the potential role of growth factors as treatment options. Hum Exp Toxicol. 2011;30:701-24.

42. Mcauley DF, Cross LM, Hamid U, Gardner E, Elborn JS, Cullen KM, et al. Keratinocyte growth factor for the treatment of the acute respiratory 
distress syndrome (KARE): a randomised, double-blind, placebo-controlled phase 2 trial. Lancet Respir Med. 2017;5:484-91.

43. Ito Y, Correll K, Schiel JA, Finigan JH, Prekeris R, Mason RJ. Lung fibroblasts accelerate wound closure in human alveolar epithelial cells through hepatocyte growth factor/c-met signaling. Am J Physiol Lung Cell Mol Physiol. 2014;307:L94-105.

44. Yogev N, Frommer F, Lukas D, Kautz-Neu K, Karram K, lelo D, et al. Dendritic cells ameliorate autoimmunity in the CNS by controlling the homeostasis of PD-1 receptor(+) regulatory T cells. Immunity. 2012;37:264-75.

45. Di Nicola M, Carlo-Stella C, Magni M, Milanesi M, Longoni PD, Matteucci P, et al. Human bone marrow stromal cells suppress T-lymphocyte proliferation induced by cellular or nonspecific mitogenic stimuli. Blood. 2002;99:3838-43.

46. Singhal E, Sen P. Hepatocyte growth factor-induced c-Srcphosphatidylinositol 3-kinase-AKT-mammalian target of rapamycin pathway inhibits dendritic cell activation by blocking IkappaB kinase activity. Int J Biochem Cell Biol. 2011:43:1134-46.

47. Wang H, Zheng R, Chen Q, Shao J, Yu J, Hu S. Mesenchymal stem cells microvesicles stabilize endothelial barrier function partly mediated by hepatocyte growth factor (HGF). Stem Cell Res Ther. 2017;8:211.

48. Shi B, Qi J, Yao G, Feng R, Zhang Z, Wang D, et al. Mesenchymal stem cell transplantation ameliorates Sjogren's syndrome via suppressing IL-12 production by dendritic cells. Stem Cell Res Ther. 2018;9:308.

49. Sato K, Yamashita N, Baba M, Matsuyama T. Modified myeloid dendritic cells act as regulatory dendritic cells to induce anergic and regulatory $T$ cells. Blood. 2003:101:3581-9.

50. Singhal E, Kumar P, Sen P. A novel role for Bruton's tyrosine kinase in hepatocyte growth factor-mediated immunoregulation of dendritic cells. J Biol Chem. 2011;286:32054-63.

51. Manning BD, Toker A. AKT/PKB signaling: navigating the network. Cell. 2017; 169:381-405.

\section{Publisher's Note}

Springer Nature remains neutral with regard to jurisdictional claims in published maps and institutional affiliations.

Ready to submit your research? Choose BMC and benefit from:

- fast, convenient online submission

- thorough peer review by experienced researchers in your field

- rapid publication on acceptance

- support for research data, including large and complex data types

- gold Open Access which fosters wider collaboration and increased citations

- maximum visibility for your research: over $100 \mathrm{M}$ website views per year

At $\mathrm{BMC}$, research is always in progress.

Learn more biomedcentral.com/submissions 